\title{
The Effect of Pressure on the Flow Behavior of Polybutene
}

\author{
Aleks Ponjavic ${ }^{1}$, Luca di Mare ${ }^{1}$ and Janet S. S. Wong ${ }^{1}$ \\ ${ }^{1}$ The Department of Mechanical Engineering, Imperial College London, London SW7 2AZ, UK \\ Correspondence to: Janet Wong (E-mail: j.wong@imperial.ac.uk)
}

\begin{abstract}
The rheology of sub-micron thick polymer melt is examined under high normal pressure conditions by a recently developed photobleached-imaging velocimetry technique. In particular, the validity and limitation of Reynolds' equation solution, which suggests a linear through-thickness profile, is investigated. Polybutene (PB) is sheared between two surfaces in a point contact. The results presented in this work suggest the existence of a critical pressure below which the through-thickness velocity profile is close to linear. At higher pressures however, the profile assumes a sigmoidal shape resembling partial plug flow. The departure of the sigmoidal profile from the linear profile increases with pressure, which is indicative of a second order phase/glass transition. The nature of the transition is confirmed independently by examining the pressure dependent dynamics of PB squeeze films. The critical pressure for flow profile transition varies with molecular weight, which is consistent with the pressure-induced glass transition of polymer melt.
\end{abstract}

\section{INTRODUCTION}

Polymer melts often experience extreme stresses during processing. When a thin film of polymer melt is subjected to a combination of high normal stresses and high shear stresses, its rheology may differ from its counterpart at ambient conditions. While it is well known that the viscosity of polymer melt can increase with normal stress, ${ }^{1}$ it can also undergo shear thinning when a shear stress is applied. ${ }^{2}$ The situation is further complicated in instances such as blow molding of multilayer films, where thin polymer melts are forced through orifices and experience a high degree of confinement. The interaction between the melt and the die surface governs the boundary condition and cannot be ignored. ${ }^{3}$ The resulting rheology is complex and hard to predict: it depends on the structure of the polymer, ${ }^{4}$ on the solid-polymer interactions ${ }^{5}$ and on the severity of the operating conditions, which is governed by temperature, pressure and shear rate. ${ }^{6}$ As a result, non-Newtonian rheology is commonly observed in polymer melt processing. Phenomena such as wall slip $^{5}$ and shear banding ${ }^{7}$ have been reported, highlighting the sensitivity of polymer rheology to experimental conditions.

Research in polymer rheology has taken a rather polarized approach. Many of the important findings on polymer rheology are based on studies with thick films, hundreds of microns and above, conducted using conventional methods such as rotational and capillary rheometry. ${ }^{8}$ One major shortcoming with these techniques comes from their limitations in applied normal stress, shear rate, and geometry. The trend in the miniaturization of production processes requires melt to be processed at normal pressures and shear rates higher than those achievable by conventional rheometric techniques to allow melt to fill submicron gaps. A high pressure rheometer, based on the diamond anvil cell, has been used to study polymer melt rheology at high pressure. ${ }^{9}$ However, the unknown, and potentially anomalous, flow of confined melt complicates the interpretation of results. ${ }^{10} \mathrm{~A}$ lot of effort has also been invested in understanding the behavior of ultra-thin (tens of nanometers or less), highly confined polymer. Research on surface effects on highly confined polymer melt rheology has been carried out using the surface 
forces apparatus. ${ }^{11,12}$ The conditions applied in these studies are nevertheless remote from any engineering significance.

Bridging the experimental and knowledge gap between these two extremes is crucial for fulfilling engineering challenges in future polymer processing. It is also fundamental, as polymer behavior at high pressure and high confinement conditions are closely linked to glassy polymer dynamics, which is one of the major research areas in polymer physics. Little is known about polymer melt rheology in the meso-scale, where continuum and molecular understanding collide. It is unclear where the boundary of the two length scales lies and how they interact and affect polymer rheology. Clearly a new approach that allows polymer rheology to be investigated across wider length scales at conditions closer to service conditions is needed. In this work, a newly developed experimental approach based on photobleached-fluorescence imaging, ${ }^{13}$ is deployed to probe local polymer melt rheology in the meso-scale, in which the melt thickness is kept below 1 micron but remains much larger than the radius of gyration of the polymer. The work focuses on how the rheology of submicron thick polymer melt is influenced by the application of normal stresses. In particular we are interested in high pressure conditions due to their engineering relevance. In these conditions, viscoelastic behavior of the polymer melt is likely to be observed.

\section{EXPERIMENTAL TECHNIQUE}

Polybutene (PB) is chosen as the model polymer melt in this study. A fluorophore, nile red, is dissolved in the melt. The melt is confined and sheared between a sphere and a flat surface while normal pressure is applied. PB in such confinement is referred to as a confined melt in this work. PB is otherwise referred to as a bulk melt. The local rheology of the confined melt is then examined by obtaining the throughthickness velocity profile, $u(z)$, using photobleached-fluorescence imaging, as detailed in the Methodology section. $u(z)$ is a direct measure of the flow property of the confined melt and hence reflects the rheology of the confined melt.

\section{Materials}

The PB used in this study is an oligomer which exhibits high viscosity despite its relatively low molecular weight. It consists mainly of repeating monomers of isobutylene ( 90\%), with the remainder consisting of monomers of 1-butene and 2-butene. At low shear rates and ambient pressure, PB is a Newtonian fluid. At the high pressures and shear rates applied in this work, it is however likely that shear thinning will occur. ${ }^{14}$

Model PB are labeled as PB920 (Sigma-Aldrich UK), PB1300 (H-300, Ineos Oligomers) and PB2300 (Sigma-Aldrich UK). Some material properties of PB are listed in Table 1.

TABLE 1 Properties of polybutene, including dynamic viscosity $\eta$, number average molecular weight $\boldsymbol{M}_{\boldsymbol{n}}$, polydispersity $\boldsymbol{M}_{\boldsymbol{w}} / \boldsymbol{M}_{\boldsymbol{n}}$, glass transition temperature $\boldsymbol{T}_{\boldsymbol{g}}$, and estimated hydrodynamic radius $\boldsymbol{R}_{\boldsymbol{H}}$.

\begin{tabular}{lccc}
\hline & PB920 & PB1300 & PB2300 \\
\hline$\eta$ at $25^{\circ} \mathrm{C}(\mathrm{Pa} \mathrm{s})^{\mathrm{a}}$ & 19.6 & 66 & 450 \\
$\eta$ at $100^{\circ} \mathrm{C}(\mathrm{Pa} \mathrm{s})^{\mathrm{b}}$ & $0.2-0.235$ & $0.65-0.7$ & $4-4.4$ \\
$M_{n}(\mathrm{~g} / \mathrm{mol})^{\mathrm{b}}$ & 920 & 1300 & 2300 \\
$M_{w} / M_{n}{ }^{\mathrm{b}}$ & - & 1.65 & - \\
$T_{g}\left({ }^{\circ} \mathrm{C}\right)^{\mathrm{b}}$ & $-68.1^{\mathrm{c}}$ & -66.9 & -64.9 \\
$R_{H}$ at $25^{\circ} \mathrm{C}(\mathrm{nm})^{\mathrm{d}}$ & 0.71 & 0.85 & 1.12 \\
\hline
\end{tabular}

a The dynamic viscosity $\eta$ at $25^{\circ} \mathrm{C}$ for PB1300 and PB2300 was measured using a Discovery Hybrid Rheometer (TA Instruments), with parallel plates at a constant shear rate of $10 \mathrm{~s}^{-1} .{ }^{b}$ Information given by suppliers. ${ }^{\mathrm{C}}$ Estimated by linear interpolation of values for PB460 and PB1290. ${ }^{d}$ Results estimated using existing small-angle X-ray scattering data ${ }^{15}$ for polyisobutylene.

Nile red was dissolved in PB at concentrations of $0.1-1 \mathrm{mM}$ by magnetic stirring. Mixing was carried out for 5 hours at a temperature of $150^{\circ} \mathrm{C}$. The fluorophore, nile red, has a $R_{H}$ of approximately $0.5 \mathrm{~nm},{ }^{16}$ which is smaller or comparable in size to the polybutene studied. No notable change in the viscosity or film thickness of PB could be observed upon the addition of nile red, indicating that the effect of nile red on the bulk rheology of PB is negligible. 
Experiments were also conducted which ensured that nile red in PB did not adsorb on the rubbing surfaces (results not shown). The diffusion coefficient of nile red in bulk PB1300 is $3.8 \times 10^{-15} \pm 1.1 \times 10^{-15} \mathrm{~m}^{2} / \mathrm{s}$ as obtained by fluorescence recovery after photobleaching. Since the pressure experienced by the polymer melt in this study increases the viscosity of the melt and hence, slows down the diffusion of nile red, the diffusion of nile red in the confined melt is assumed to be negligible within the experimental time frame. The amount of heat generated due to the applied methodology and due to shear heating has been estimated based on established models, and is shown to be negligible. ${ }^{13}$

\section{Methodology}

Photobleached-fluorescence imaging, detailed in ${ }^{17}$, has been modified and applied to this work. Briefly, the fluorophore-doped polymer melt is confined by a rubbing device. The rubbing device consists of a rotating sphere and a stationary glass flat as shown in Figure 1 . The setup results in the melt being confined in a circular point contact. When high normal pressure is applied through the sphere, the film thickness of the confined melt is roughly uniform across most of the contact. The rubbing device is placed on an inverted microscope. The melt gives a homogeneous glow when it is observed by epi-fluorescence microscopy through the glass flat.

A through-thickness photobleached column, which has lower fluorescence intensity than the rest of the confined melt, is created by exposing the confined melt, at a particular position within the contact, to a high energy, highly focused laser (photobleaching beam) for a short period of time. This column initially appears as a circular spot when observed with a microscope on the $x-y$ plane. As the melt is sheared, the spot is distorted. Experimental results are in the form of image sequences taken by an EMCCD camera that show the shape evolution of the spot. A typical sequence consists of 20 images taken with an exposure and a cycle time of $1 \mathrm{~ms}$ and $1.5 \mathrm{~ms}$ respectively. This corresponds to 30 ms per sequence. Synchronized averaging ${ }^{18}$ is applied to increase the signal-to-noise ratio of the images, and hence the resolution of the developed method. The resulting spatial $x, y$, and $z$ resolutions are $30 \mu \mathrm{m}, 3 \mu \mathrm{m}$ and $\frac{h}{12}$ ( $h$ is the thickness of the confined melt) respectively which are determined by the size of the photobleached column and the film thickness.

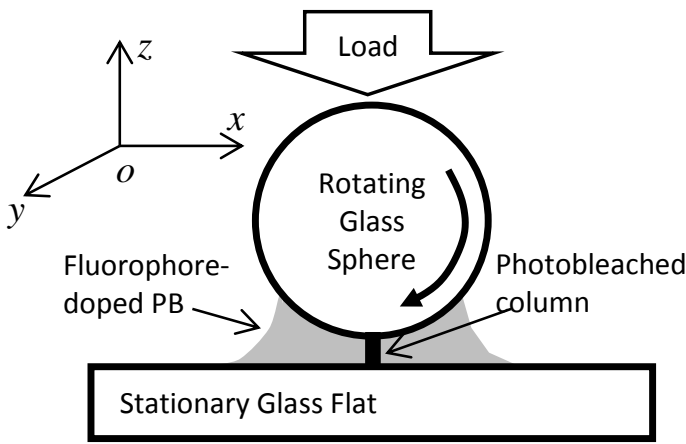

FIGURE 1 Schematic showing the experimental conditions employed in this work. Experimental observation is carried out on the $x-y$ plane and the projected photobleached column appears initially as a circle. The through-thickness velocity profile in the $z$-direction is constructed based on the evolution of this circle.

The experimental results can be used to determine the through-thickness velocity profile, $u(z)$, at a particular position $(x, y)$ in the contact using an algorithm developed in ${ }^{13}$. The algorithm estimates $u(z)$ with the assumptions that the diffusion of nile red in the confined melt is negligible and that the melt consists of infinitesimal layers of fluids parallel to the stationary glass flat, where $z$ is the distance between the position of a layer of melt and the glass flat. Simulated image sequences are produced based on the reconstructed velocity profile. The reconstructed profile is optimized through an iteration process by minimizing the difference in experimental results and the simulated results. ${ }^{13}$

Instead of the steel sphere employed in ${ }^{13}$, a glass sphere is used to shear the PB melt in this work. When a steel sphere is used as one of the 
rubbing surfaces, the reflection of the photobleaching beam by the reflective steel surface can induce interference within the thickness of the polymer melt. ${ }^{19}$ Destructive interference fringes may form and the positions of these fringes depend on the thickness of the melt. This interference effect can be incorporated into the algorithm for profile reconstruction. ${ }^{19}$ The results in ${ }^{13}$ have been reanalyzed, assuming the existence of destructive interference fringes. While the conclusion that the flow of PB deviates from noslip Couette flow description under a high normal stress still holds, the uncertainty of the reconstructed profiles, in particular the velocity of the melt near the two rubbing surfaces, increases substantially. As a result, the existence of interfacial slip cannot be conclusively ascertained. To avoid this complication, a borosilicate glass sphere is used in this study. Control experiments by laserinduced fluorescence imaging (LIF) show that the fluorescence intensity with this new setup varies linearly through the thickness of melt in the point contact (not shown), confirming that the interference effect is negligible with the setup used in this work.

Borosilicate glass is weakly phosphorescent, with a life time of the order of milli-seconds. To eliminate this phosphorescent signal, a control image sequence for every applied experimental condition is acquired without illumination after photobleaching has been carried out. This gives a control sequence containing only phosphorescent signal from glass. All image sequences used to determine $u(z)$ have been corrected by their corresponding control sequences to ensure only signal from the confined melt is used for $u(z)$ reconstruction.

All experiments were performed at room temperature $\left(25 \pm 0.5{ }^{\circ} \mathrm{C}\right)$. A normal load and a shear stress were applied by the rubbing device. The shear stress was applied in the $x$-direction. The normal pressure (in the $z$-direction) experienced by the melt is estimated by Hertzian contact mechanics with the Young's Modulus and Poisson's ratio of glass set to 70 GPa and 0.2 respectively. This results in a parabolic normal stress distribution where the stress reaches a maximum at the centre of the contact. The diameter of the contact increases with the applied normal pressure and ranges between $200 \mu \mathrm{m}-400 \mu \mathrm{m}$. The thickness of the melt, $h$, which is governed by the experimental conditions and the local viscosity of the melt, is measured by laser-induced fluorescence imaging. ${ }^{20}$ All velocity profiles were obtained at the centre of the point contact where the gradient of the normal stress is approximately zero. As the flow of confined $\mathrm{PB}$ at $y=0$, including at the centre of the contact, is dominated by flow in the $x$-direction due to symmetry, the one-dimensional Reynolds equation can be applied in this work. Compliance of the test apparatus means that rotation of the glass sphere may induce a maximum drift in the contact position of 0.1 $\mu \mathrm{m} / \mathrm{s}$. This is negligible compared to the rotational speed of the sphere and is neglected. Results presented are averages of at least 100 image sequences. The ranges of normal pressure and surface speed applied are 150 $500 \mathrm{MPa}$ and $360-720 \mu \mathrm{m} / \mathrm{s}$ respectively.

\section{RESULTS AND DISCUSSIONS}

The one-dimensional Reynolds equation is frequently used to describe the flow of a thin film of fluid in isothermal conditions. Assuming that the no-slip boundary condition applies, a common solution, given in equation (1), shows that a fluid with viscosity, $\mu$, and a film thickness, $h$, being sheared in the $x$-direction has a velocity profile, $u(z)$, given by a combination of a pressure-driven Poiseuille term, $\left(z^{2}-z h\right)$; and a shear driven Couette term, $\left(\frac{z}{h}\right)$.

$u(z)=\frac{1}{2 \mu} \frac{\partial P}{\partial x}\left(z^{2}-z h\right)+U \frac{z}{h}$ 
In the simplest case, where $\mu$ is large (i.e. highly viscous) and/or $\frac{\partial P}{\partial x}$ is zero (i.e. at maximum $P$ ), pressure driven flow is eliminated and only linear Couette flow remains:

$u(z) \approx U \frac{z}{h}$

Equation (1) suggests that the flow of confined $\mathrm{PB}$ at the centre of the contact, where there is maximum pressure and $\frac{\partial P}{\partial x}$ is zero, should obey equation (2). Two questions are addressed in this work. Firstly, can a linear $u(z)$, as shown in equation (2) describe the flow, and hence the rheology of confined PB experiencing high normal stress? If so, secondly, what condition would render this description inapplicable?

To answer these questions, $u(z)$ was obtained for PB at various applied normal stresses, $P$, and speeds of the glass sphere, $U$. The results obtained suggest that the rheology of confined PB is dominated by $P$, rather than $U$. Depending on the applied normal pressure, two types of through-thickness profiles were observed which are detailed below.
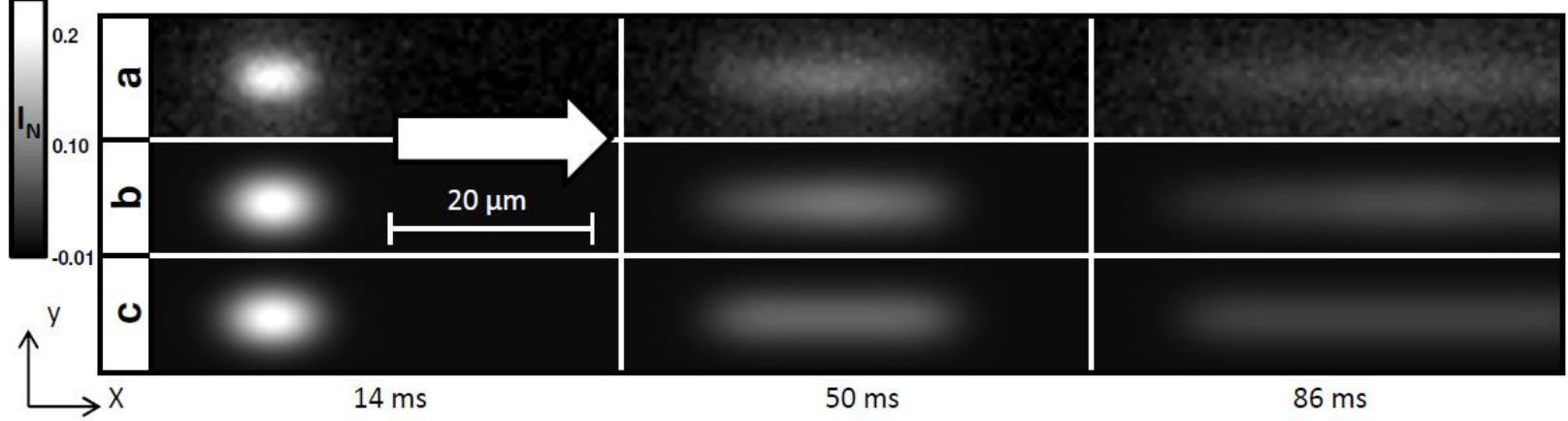

FIGURE 2 Snapshots showing the shape evolution of the photobleached shot as PB2300 was sheared at $\boldsymbol{P}=333$ MPa and $\boldsymbol{U}=360 \mu \mathrm{m} / \mathrm{s}$ : (a) The experimental results are compared with (b) simulated results from the profile reconstruction algorithm and (c) Couette flow prediction. The grayscale value represents the relative normalized intensity distribution (the degree of photobleaching) as previously defined. ${ }^{13}$ White and black in the grayscale image correspond to low and high intensity respectively. The thickness of the confined polymer melt was $\boldsymbol{h}=630$ $\mathrm{nm}$. The white arrow indicates the direction of the flow.

\section{Low pressure condition}

As outlined in the Methodology section, a profile reconstruction algorithm is used to obtain $u(z)$ by generating simulated results that match well with experimental results. Figure 2(a) shows a sequence of images obtained with PB2300 at $P=333 \mathrm{MPa}$. The corresponding simulated sequence based on $u(z)$ obtained with the developed algorithm, and the image sequence, assuming the melt obeys Couette prediction, are presented in Figure 2(b) and Figure 2(c) respectively.

The photobleached spot is stretched substantially over time as a result of the applied shear stress. There is a good match between experimental (Figure 2(a)) and simulated results (Figure 2(b)) as indicated by the goodness of fit,
$R^{2}=0.987$. This confirms that the developed algorithm has successfully reconstructed the through-thickness velocity profile giving rise to experimental observations. Both the experimental and simulated results closely resemble the intensity distribution based on Couette flow (Figure 2(c)).

The reconstructed $u(z)$ (white circles) based on results in Figure 2 is compared to the Couette flow profile (dashed line) in Figure 3. Recall that the glass flat, where $\frac{z}{h}=0$, is stationary, and the glass sphere, located at $\frac{z}{h}=1$, was rotating at $U=360 \mu \mathrm{m} / \mathrm{s}$. Two observations are made. Firstly, at $\frac{z}{h}$ equals 0 and $1, \frac{u(z)}{U}$ equals 0 and 1 respectively, i.e. the melt at the proximity of the rubbing surfaces travels at the same speed as the rubbing surfaces. Hence the flow of the PB 
obeys the no-slip boundary condition. This is consistent with studies showing that PB sticks to the glass surface. ${ }^{21}$

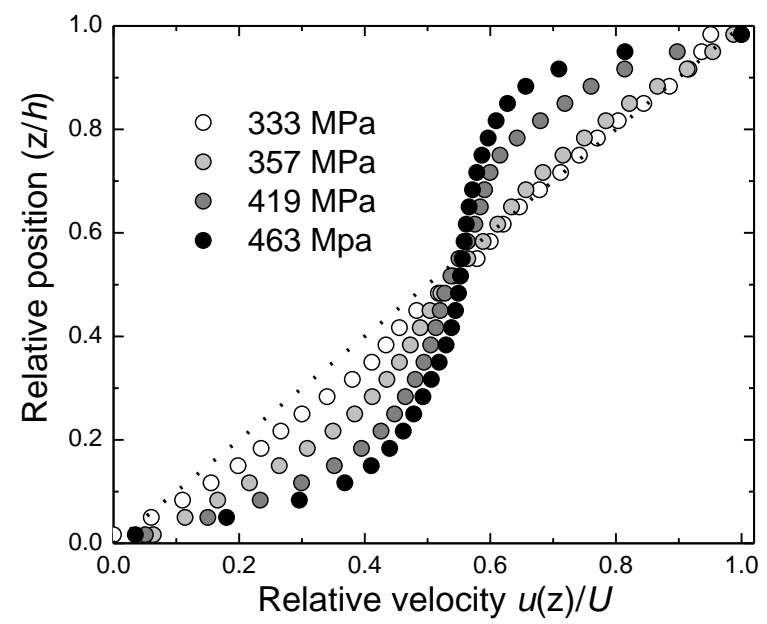

FIGURE 3 Through-thickness velocity profile, $\boldsymbol{u}(\mathbf{z})$, obtained for PB 2300 melt with $\boldsymbol{U}=360 \mu \mathrm{m} / \mathrm{s}$. The dash line corresponds to the linear Couette flow profile.

Secondly, $u(z)$ at $P=333 \mathrm{MPa}$ (white circle in Figure 3 ) roughly follows the Couette flow profile (dash line), with the relative difference, quantified by the mean velocity, being $5 \%$. This deviation can be due to the existence of pressure driven flow in the melt. The centre of a stationary circular point contact, similar to the contact geometry used in this study, should experience the maximum pressure, hence $\frac{\partial P}{\partial x}$ is zero. However, the application of shear motion can shift the normal pressure distribution; as a result the position of maximum $P$ can be slightly off-centre. This could result in a nonzero pressure-driven component adding to the otherwise linear flow profile, hence the observed slightly bulged profile.

The normalized shear rate at various $z$ can be extracted from the through-thickness velocity profile. An increase in the slope $\frac{d z}{d u}$ corresponds to a reduction in shear rate. The resulting $u(z)$ at the low normal pressure condition (white circles in Figure 3 ) shows that the shear rate experienced by the melt is relatively constant across the thickness of the melt.

\section{High pressure condition}

The shape evolution of the photobleached spot in PB2300 when applied normal stress was 463 $\mathrm{MPa}$, is presented in Figure 4. It differs significantly from experiments conducted at lower normal pressures (Figure 2). At $P=463$ $\mathrm{MPa}$, the photobleached spot shows only a very small amount of stretching, as opposed to the highly elongated spot shown in Figure 2 . This suggests that linear flow is not observed in the case of high normal pressure. The reconstructed $u(z)$, based on images in Figure 4, is presented in Figure 3 (black circles). It exhibits a sigmoidal shape and violates the Couette prediction (dashed line).

The $u(z)$ profile at $P=463 \mathrm{MPa}$ is symmetric about $\frac{z}{h}=0.5$. It also shows that the no-slip boundary condition holds at the interfaces. Contrary to the $u(z)$ obtained at $P=333 \mathrm{MPa}$, the local normalized shear rate when $P=463$ $\mathrm{MPa}$ is not constant across the thickness of the melt, but is rather a function of $\frac{z}{h}$. Starting from the stationary glass flat $\left(\frac{z}{h}=0\right)$, the melt experiences a decreasing shear rate until $\frac{z}{h}$ reaches roughly 0.3 . The shear rate of the confined melt in the central region of the film, between $\frac{z}{h}=0.3$ and 0.7 , is roughly constant, and low. From $\frac{z}{h}=0.7$, the normalized shear rate increases again and the melt reaches the highest shear rate at the glass sphere surface for $\frac{z}{h}=1$. A near zero shear rate, i.e. a vertical $u(z)$, observed between $\frac{z}{h}=0.3$ and 0.7 suggests the existence of plug flow at the centre of the melt. This plug is surrounded by melt that sustains much higher shear rates. The fact that different parts of the melt exhibit different flow behavior supports the existence of structural and/or rheological heterogeneity in the melt when high normal pressure is applied. This phenomenon is commonly referred to as inhomogeneous shear, and similar velocity profiles have been observed for flows of heterogeneous material such as foams ${ }^{22}$ and entangled polymers. ${ }^{23}$ 


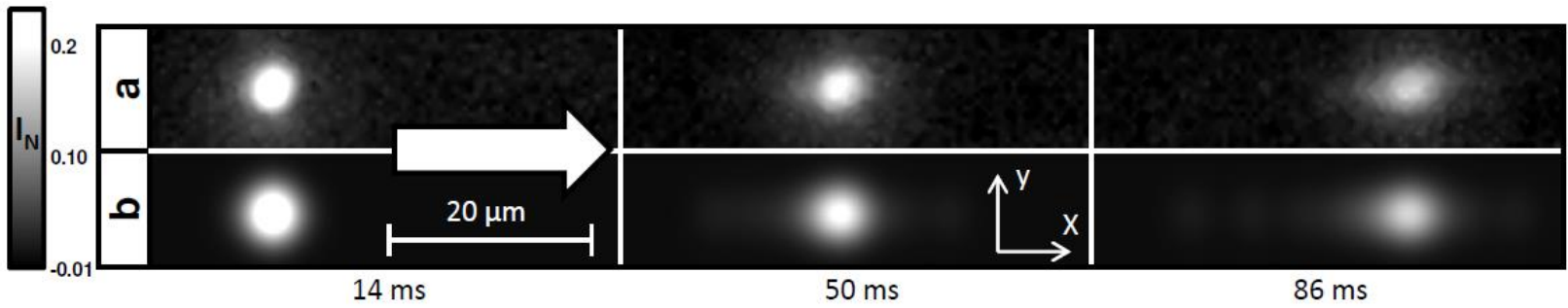

FIGURE 4 Snapshots showing the shape evolution of the photobleached spot as PB2300 was sheared at $P=463$ MPa and $U=360 \mu \mathrm{m} / \mathrm{s}$ : (a) The experimental results are compared with (b) the simulated results obtained using the profile reconstruction algorithm. The grayscale value represents the relative normalized intensity distribution (the degree of photobleaching) as previously defined. ${ }^{13}$ White and black in the grayscale image correspond to low and high intensity respectively. The thickness of the confined polymer melt was $h=830 \mathrm{~nm}$. The white arrow indicates the direction of the flow.

\section{Pressure sensitive rheological transition}

Two types of through-thickness velocity profiles have been presented. At $P=333 \mathrm{MPa}, u(z)$ resembles a Couette profile with a small contribution from pressure driven flow. At $P=$ $463 \mathrm{MPa}$, a sigmoidal profile is observed with the centre of the melt showing plug flow behavior, while the adjacent melt next to the rubbing surfaces experiences much higher shear rates. On first consideration this observed change in PB rheology may be due to (1) the imposed geometric confinement, and (2) the applied normal pressure. Previous observations suggest that only geometric confinement in the nanoscale affects the dynamics of polymer melt. ${ }^{12,24}$ The thickness of the polymer melt in this study, however, is in the submicron range which is much larger than the size of the polymer. Thus it is likely that the effect of geometric confinement only applies to polymer in proximity of the rubbing surfaces. Hence geometric confinement, at least in the length scale applied in this work, may only play a secondary role in the rheology of the confined and sheared PB. The surface effect on the $u(z)$ will be discussed in the next session.

Interestingly, thickness measurements conducted with laser-induced fluorescence imaging show that the thickness of the melt at the centre of the point contact is insensitive to applied normal pressure when pressure is low, as predicted from elastohydrodynamic lubrication theory. ${ }^{25}$ Once the applied pressure is above the critical value, the thickness of the melt increases with normal pressure. The change in thickness alone, while capable of reducing the flow velocity of the melt, however, cannot cause the observed sigmoidal velocity profile. This abrupt change in thickness response with respect to pressure coincides with the occurrence of sigmoidal velocity profiles, suggesting both events are possibly related and are pressure-induced phenomena.

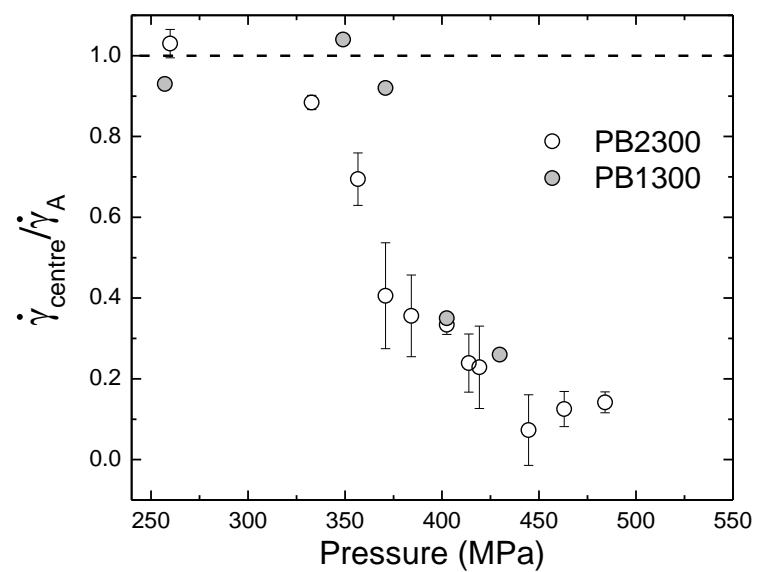

FIGURE 5 The relationship between central shear rate, $\dot{\gamma}_{\text {centre }}$, and the normal pressure applied. $\dot{\gamma}_{\text {centre }}$ is normalized by the apparent shear rate, $\dot{\gamma}_{\boldsymbol{A}}=\frac{\boldsymbol{U}}{\boldsymbol{h}}$. The dash line represents $\dot{\gamma}_{\text {centre }}$ for the Couette condition.

Viscosity and dynamics of polymer have been shown to be pressure dependent. ${ }^{1,26}$ The sensitivity of polymer dynamics to applied pressure can also be enhanced by geometry confinement. ${ }^{12}$ Through-thickness velocity profiles obtained at intermediate normal 
pressures are shown in Figure 3 (gray circles). They conform to the sigmoidal profile seen at $\boldsymbol{P}$ $=463 \mathrm{MPa}$. Figure 5 correlates the shear rate at the centre of the melt, $\dot{\gamma}_{\text {centre }}$, i.e. the slope of $\boldsymbol{u}(\mathbf{z})$ at $\frac{\boldsymbol{z}}{\boldsymbol{h}}=0.5$, with the applied normal pressure. Two distinct regions can be identified, with high and low $\dot{\gamma}_{\text {centre }}$ observed at low and high pressures respectively. This alludes to an existence of a critical normal pressure which governs the flow behavior of PB.

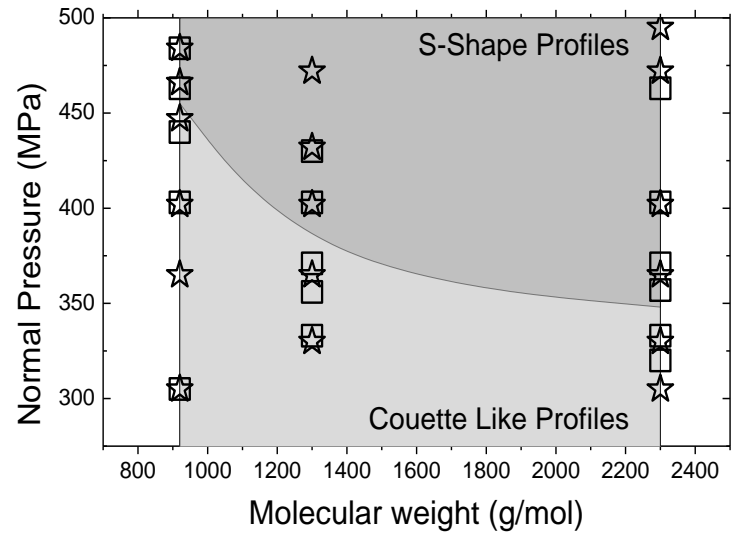

FIGURE 6 The correlation between the applied normal pressure, $P$, and the type of throughthickness velocity profile obtained at $U=360 \mu \mathrm{m} / \mathrm{s}$. Light grey and dark grey area indicate regions where Couette-like and sigmoidal profiles were obtained respectively. Star: results from rotating glass sphere against stationary sapphire flat; square: results from rotating glass sphere against stationary glass flat.

This pressure-induced transition in the rheological behavior of the PB melt was investigated for PB920, PB1300 and PB2300 and the results are summarized in Figure 6 . All three PB melts show a transition from Couette-like profiles to sigmoidal profiles at a critical value of pressure. The critical pressure values are similar between experiments conducted with a stationary sapphire flat (Figure 6 stars) and with a stationary glass flat (Figure 6 squares). The critical pressures are about $450 \mathrm{MPa}, 390 \mathrm{MPa}$, and $355 \mathrm{MPa}$ for PB920, PB1300 and PB2300 respectively. An increase in molecular weight increases the longest relaxation time of the polymer, which is responsible for an increase in viscosity and also a reduction in glass transition temperature. ${ }^{27,28}$ Hence, this variation in critical pressure is consistent with a reduction in the glass transition temperature, and hence an increase in glassy tendency, with increasing molecular weight. This validates that the rheology of PB observed in this work is mainly determined by the applied normal pressure.

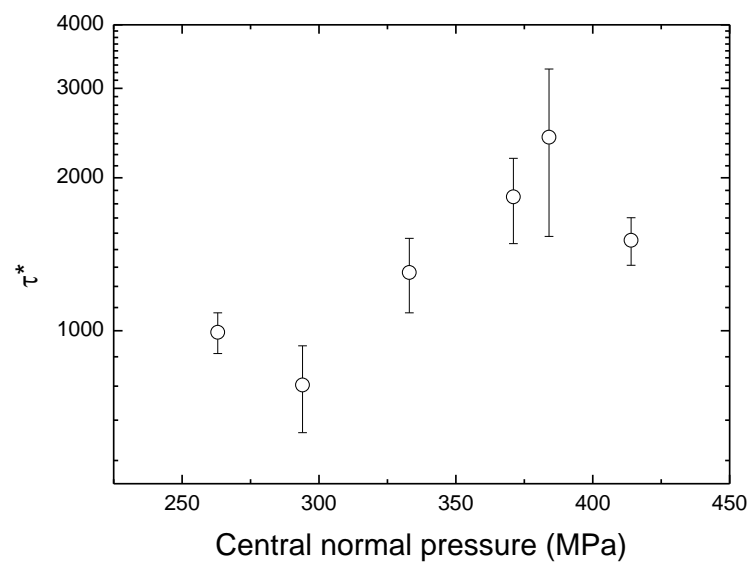

FIGURE 7 The relationship between the nondimensional characteristic decay time for film thickness reduction at various applied normal stresses obtained by squeezing PB2300 between a stationary glass sphere and a stationary glass flat.

The effect of normal stress on the dynamics of PB2300 is further investigated by studying how the thickness of PB2300 film changes with time between a stationary glass sphere and a stationary flat under various static normal stresses. Such a setup creates a squeeze film. The decrease in the film thickness over time can be described by a characteristic decay time $\boldsymbol{\tau}^{29}$ which can be non-dimensionalized by the viscosity and the pressure giving $\tau^{*} .^{30}$ The variation of $\tau^{*}$ with pressure is shown Figure 7. Two non-dimensional characteristic decay times can be identified, one at around 1000 and the other at around 2500 for central normal pressures below $300 \mathrm{MPa}$, and above $370 \mathrm{MPa}$ respectively. The transition from short to long $\tau^{*}$ occurs gradually, suggesting a second order transition, which matches the nature of a glass transition. The midpoint of the transition lies at about $340 \mathrm{MPa}$ which coincides with the normal pressure that induces a transition in PB2300 from linear flow to sigmoidal plug flow, as shown in Figure 6. Hence this supports that the onset of plug flow in the confined PB melt is a 
second order transition induced by increased normal pressure.

The results presented strongly suggest that a pressure-induced glass transition causes the reported phenomena. Pressure-induced structural changes, which include glasstransition, have been observed in small molecule liquids and polymers. ${ }^{31-33}$ Indeed PB is a well-known glass former, with a glass viscosity of $10^{7} \mathrm{~Pa} \mathrm{~s}^{14}$ The effect of normal pressure on the viscosity of a liquid, $\eta$, can be described approximately by the Barus equation

$\eta=\eta_{0} e^{\alpha P}$

where $\eta_{0}$ is the low shear rate viscosity and $\alpha$ is the pressure-viscosity coefficient. Table 2 lists the viscosity of PB estimated with equation (3) at pressures applied in this work, assuming $\alpha=$ $30 \mathrm{GPa}^{-1}$ for all three PB melts. The viscosity of $P B$ at the critical pressure is about the same order of the glass viscosity obtained in the literature. ${ }^{14}$ Hence the condition that promotes plug flow in $P B$ coincides with the glass transition of PB. As the viscosity of the PB glass increases, $\dot{\gamma}_{\text {centre }}$ decreases.

TABLE 2 Viscosity of PB under various normal pressure estimated using the Barus equation.

\begin{tabular}{|c|c|c|c|}
\hline & \multicolumn{3}{|c|}{$\operatorname{Viscosity~}^{\mathrm{a}}\left(\times 10^{7} \mathrm{~Pa} \mathrm{~s}\right)$} \\
\hline & PB920 & PB1300 & PB2300 \\
\hline \multirow{2}{*}{$\begin{array}{l}\text { Below critical } \\
\text { pressure }\end{array}$} & 0.32 & 0.24 & 0.77 \\
\hline & (400 MPa) & (350 MPa) & (325 MPa) \\
\hline \multirow{2}{*}{$\begin{array}{l}\text { At the critical } \\
\text { pressure }\end{array}$} & 1.43 & 0.80 & 1.90 \\
\hline & (450 MPa) & (390 MPa) & (355 MPa) \\
\hline \multirow{2}{*}{$\begin{array}{l}\text { Above critical } \\
\text { pressure }\end{array}$} & 4.09 & 2.27 & 7.32 \\
\hline & (485 MPa) & (425 MPa) & (400 MPa) \\
\hline
\end{tabular}

\section{Formation of constricted plug flow}

If $\mathrm{PB}$ at the centre of the point contact has indeed undergone a glass transition due to the applied normal pressure and is solid-like, the question remains of why the development of plug flow is constricted to the region between $\frac{z}{h}$ equals 0.3 and 0.7 . In other words, what is preventing the formation of a through-thickness plug? The spatial-resolution in the $z$-direction of the applied methodology has been estimated to be no less than $0.1 \mathrm{~h}$. Hence the current methodology would be capable of capturing the existence of a fully developed through-thickness plug. It is possible that the constriction of plug flow might be caused by (1) surface heating; and (2) surface induced structural changes.

The estimated heat due to frictional heating would result in an increase of $0.01 \mathrm{~K}^{13}$ The temperature rise due to photobleaching is $3.5 \mathrm{~K}$ and the heat is dissipated in $1 \mathrm{~ms}$. Hence both heat sources are negligible in this study. ${ }^{13}$ For argument's sake, the potential effect of temperature on $u(z)$, can be investigated by representing the flow profile as a small perturbation of a Couette flow. $u(z)$ is originally linear under the applied experimental conditions, and the temperature of both rubbing surfaces is equilibrated at $T^{*}$. A small perturbation, $\epsilon$, which represents the dependence of the viscosity on temperature, i.e. $\left.\epsilon \propto \frac{\partial \eta}{\partial T}\right|_{P}$, is then introduced. Applying the stick boundary conditions and that temperature of confined melt at the interface is $T^{*}$ gives a symmetric through-thickness temperature distribution where the temperature is highest at $\frac{z}{h}$ equals 0.5 . If $\epsilon$ is negative, i.e. the viscosity of PB decreases with increasing temperature, the confined PB should have low viscosity, hence high shear rate at $\frac{z}{h}=0.5$. This contradicts experimental results presented in Figure 3 which shows that the shear rate is lowest at $\frac{z}{h}=$ 0.5 . While localized heating may occur adjacent to the interface and result in a reduction in viscosity of the melt next to the rubbing surfaces, giving rise to the $u(z)$ observed in this study, the origin of such localized heating remains unclear. Temperature alone is unlikely to cause the observed constricted plug flow. 
Surface induced structural changes has been observed in polymers. ${ }^{34,35}$ The conformation and dynamics of polymers in the proximity of a surface depend on the interaction energy between polymer chains and the surfaces, $E, 5,36$ and also on the topography of the surfaces. ${ }^{38}$ As a result, the properties of polymers in the surface affected zone (SAZ) and of those unaffected can be very different. The extent to which this surface effect propagates into the bulk is a subject of controversy. Experiments on glass transition of supported and free standing polymer films suggest that the thickness of this SAZ is strongly influenced by $E$ and the molecular weight of the polymer, and can range from 2 to $10 R_{g}$ (radius of gyrations). ${ }^{36,37}$ Force measurements under quasi-static condition also support that the SAZ of polymer melt confined by mica surfaces is small, of the order of $2 R_{g}{ }^{38}$ However, the rheological effect of the SAZ can extend much further into the bulk, as far as hundreds of $\mathrm{nm}$, when the applied shear rate is high. ${ }^{39}$ The thickness of the SAZ is likely to be governed by the structure of the confined melt, and the correlation between the relaxation time of polymer in the SAZ and the apparent shear rate. The glassy nature of the confined $\mathrm{PB}$ in this study exemplifies this shear rate effect, giving rise to a thick SAZ. The properties of polymers in the $S A Z$, due to their unique conformations, can be anisotropic. This anisotropy in SAZ can also promote through-thickness heterogeneous response. Hence the observed constricted plug flow can be a consequence of surface induced structural changes and hence viscosity inhomogeneity in the confined PB. Further work is required to clarify the relative importance of these factors in governing the rheology of confined polymer melt.

\section{CONCLUSIONS}

The rheology of polymer melt confined in a point contact under high normal pressures was investigated by obtaining the through-thickness velocity profile. Contrary to common belief, a linear Couette velocity profile can only be facilitated when the melt experiences a sufficiently low normal pressure. As the normal pressure increases above a critical value, constricted plug flow, together with an abrupt increase in film thickness, is observed. This Couette flow-plug flow transition is caused by a pressure-induced glass transition, where the viscosity of part of the melt has reached the glass viscosity and hence becomes solid-like. However surface-induced polymer structural changes, together with applied high shear rate, may generate structural inhomogeneity within the melt and prevent the development of complete through-thickness plug flow, despite the application of high normal pressure.

The observed constricted plug flow has significant consequence in polymer processing, in particular for blow molding of multi-layer films. The rheological heterogeneity can give rise to anisotropy in both molecular alignment and the residual stress distribution which will impact on the mechanical strength and permeability of the polymer films. The observed transitional flow behavior due to the exerted normal pressure has strong implication to the rheology of polymer melt in a point contact. The parabolic normal pressure distribution in the point contact causes the melt close to the edge of the contact to experience much lower pressure than that at the centre. The existence of a critical normal pressure for the flow transition of PB would imply that PB flow in a point contact is spatially heterogeneous. This conjecture can be examined by obtaining $u(z)$ at various positions along $y=0$ and is currently underway.

\section{ACKNOWLEDGEMENTS}

This work is supported by EPSRC platform grant no. EP/G026114/1 and EPSRC Grant no. $E P / J 008141 / 1$. A.P. was sponsored by EPSRC DTA studentship. The authors would like to thank Johan Guegan for interferometry measurements and Hugh Spikes, Daniele Dini, and David Heyes for fruitful discussions. 


\section{REFERENCES AND NOTES}

$1 \quad$ N. Hirai, H. Eyring, J. Polym. Sci. 1959, 37, 5170

2 M. M. Cross, Rheol. Acta 1979, 18, 609-614.

3 N. E. Kissi, L. Léger, J. M. Piau, A. Mezghani, J. Non-Newtonian Fluid Mech. 1994, 52, 249-261.

4 R. Everaers, S. K. Sukumaran, G. S. Grest, C. Svaneborg, A. Sivasubramanian, K. Kremer, Science 2004, 303, 823-826.

5 S. G. Hatzikiriakos, Prog. Polym. Sci. 2012, 37, 624-643.

6 T. Sedlacek, M. Zatloukal, P. Filip, A. Boldizar, P. Saha, Polym. Eng. Sci. 2004, 44, 1328-1337.

7 S. Bair, F. Qureshi, W. O. Winer, J. Tribol. 1993, $115,507$.

8 J. Meissner, Annu. Rev. Fluid Mech. 1985, 17, 4564.

9 S. Bair, Proc. Inst. Mech. Eng., Part J 2001, 215, 223-233.

P. D. Olmsted, Rheol. Acta 2008, 47, 283-300.

11 J. Van Alsten, S. Granick, Macromolecules 1990, $23,4856-4862$.

12 G. Luengo, F.-J. Schmitt, R. Hill, J. Israelachvili, Macromolecules 1997, 30, 2482-2494.

13 A. Ponjavic, M. Chennaoui, J. S. S. Wong, Tribol. Lett. 2013, 50, 261-277.

14 S. Bair, J. Non-Newtonian Fluid Mech. 2001, 97, 53-65.

15 M. Osa, F. Abe, T. Yoshizaki, Y. Einaga, H. Yamakawa, Macromolecules 1996, 29, 23022308.

N. Sarkar, K. Das, D. N. Nath, K. Bhattacharyya, Langmuir 1994, 10, 326-329.

17 B. P. Mosier, J. I. Molho, J. G. Santiago, Exp. Fluids 2002, 33, 545-554.

18 S. Braun, Mech. Syst. Sig. Process. 2011, 25, 1087-1102.

19 J. Sugimura, M. Hashimoto, Y. Yamamoto, Tribol. Ser. 2000, 38, 609-617. 2010, 43, 1960-1969.

F. Guo, X. M. Li, P. L. Wong, Tribol. Int. 2011, $46,22-29$.

G. Katgert, M. Möbius, M. van Hecke, Phys. Rev. Lett. 2008, 101, 3-6.

P. Tapadia, S. Q. Wang, Phys. Rev. Lett. 2006, 96, $1-4$.

H. W. Hu, S. Granick, Science 1992, 258, 13391342.

R. Gohar, Elastohydrodynamics; World Scientific Publishing Company, 2001.

J. S. S. Wong, L. Hong, S. C. Bae, S. Granick, J. Polym. Sci. Part B: Polym. Phys. 2010, 48, 25822588.

H. Toratani, K. Takamizawa, Polym. J. 1994, 26, 797-803.

R. Casalini, C. Roland, Phys. Rev. B 2002, 66, 180201.

W. Hardy, I. Bircumshaw, Proc. R. Soc. Lond. A 1925, 108, 1-27.

R. F. Lu, J. R. Lin, Tribol. Int. 2007, 40, 125-131.

P. Zoller, D. Walsh, Standard Pressure-VolumeTemperature data for polymers; CRC Press, 1995.

I. Noda, G. M. Story, C. Marcott, Vib. Spectrosc. 1999, 19, 461-465.

A. Reiser, G. Kasper, S. Hunklinger, Phys. Rev. B 2005, 72, 094204.

L. Delle Site, C. Abrams, A. Alavi, K. Kremer, Phys. Rev. Lett. 2002, 89, 156103.

K. A. Smith, M. Vladkov, J. L. Barrat, Macromolecules 2005, 38, 571-580.

C. B. Roth, K. L. McNerny, W. F. Jager, J. M. Torkelson, Macromolecules 2007, 40, 2568-2574.

J. Forrest, K. Dalnoki-Veress, J. Dutcher, Phys. Rev. E 1997, 56, 5705-5716.

J. N. Israelachvili, S. J. Kott, J. Chem. Phys. 1988, 88,7162 .

J. N. Israelachvili, S. J. Kott, L. J. Fetters, J. Polym. Sci. Part B: Polym. Phys. 1989, 27, 489502. 


\section{GRAPHICAL ABSTRACT}

Aleks Ponjavic, Luca di Mare and Janet S. S. Wong

The Effect of Pressure on the Flow Behavior of Polybutene

The rheology of confined polymer melt experiencing high normal pressure is investigated by obtaining through-thickness velocity profiles of polybutene sheared between two glass surfaces. These profiles are linear (Couette flow) at low pressure. They become sigmoidal (constricted plug flow) when a critical pressure is reached. This transition is shown to be a result of pressure-induced glass transition of polybutene. The sigmoidal shaped profiles signify that the through-thickness viscosity of the highly stressed melt is heterogeneous.

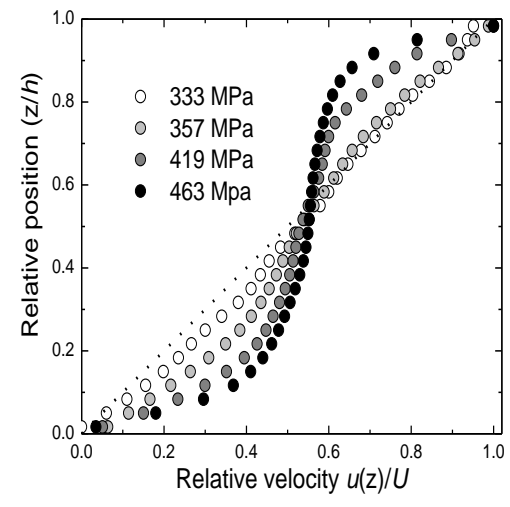

